\title{
An Exploratory View on Congregating God and Science through Variegating Perspectives
}

\author{
Mr. Jaswin Kumar N. R. \\ Class XI \\ Stanes Anglo Indian Higher Secondary School \\ Coimbatore, Tamil Nadu, India
}

\begin{abstract}
Science is often associated with atheistic ideologies. The field of science is thought to be as one of the disciplines, wherein only rational and logical theories are often applicable. Moreover, anything that cannot be explained in terms of logical scientific theories or formula is termed as supernatural or irrational. This has also created a stereotypic notion among people that whoever is a patron of scientific ideas ought to be an atheist. Some scientists have been a fuel to this stereotype by openly embracing atheism. Furthermore, rationalism has gotten us far in the field of science. However, the ground reality is that the answers to seemingly impossible-tosolve questions cannot be discovered with logic alone. Hence, we refuse to believe in something, which is not scientific. Also, we may consider things, which we think as 'beyond science' to make further developments within it. The reason being, it enables the mind to embrace creativity and look at various possibilities from different perspectives. This perspective of scientific thinking might even help us to resolve paradoxes and provide answers to questions like 'Does God Exist?' Keeping these aforementioned notions, this conceptual paper overlooks on scientific theories with an exploratory view from a variegating perspective by substantiating the 'String Theory' concepts to understand the possibility of existence of higher dimensions (up to $10^{\text {th }}$ dimension) and facilitates to explain the existence of God.
\end{abstract}

Keywords: Higher Dimensions, Supernatural, Universe, God, String Theory.

\section{Introduction}

As per various religions and beliefs, "God" is an omnipresent, omnipotent and omniscient being, capable of creation and destruction of the Universe and everything in it. God is feared and worshipped because of his "supernatural abilities". There are many myths and stories which speak of God doing "miracles" and helping humans accomplish seemingly impossible tasks. In other ancient societies the stories of extra-terrestrial beings establishing contact with humans is depicted. Surely, humans are not capable of such supernatural activities. If the 
claims are true, it has to be someone (or something) who has powers beyond the limits of our Universe. All of this points to one thing - higher dimensions. The dimensions higher than one we live in have proposed in many physical and mathematical theories by several scientists across the world, ever since Einstein in his theories of General and Special Relativity proposed that gravity is actually a curvature in the space-time continuum (Einstein, 1921). It has been since then; scientists have built upon his theories, by attempting to propose a "Theory of Everything" which shall put together every single theory that explains a part of the functioning of this universe, to make a theory that explains our universe and the laws that guide it in one single theory. This has led to the proposition of "Multiverses" and "Higher Spatial Dimensions" as an attempt to support the proposed theories. "String Theory" is a collection of theories which attempt to explain the functioning of the universe from the quantum level, by explaining the functioning of the most fundamental of all particles - the string. However, this theory requires 9 spatial dimensions and 1 temporal dimension (time) to hold true. Each higher dimension includes more and more entities of this Universe and even parallel Universes. This paper explores the possibility of a being existent in higher dimensions, and the control it has over its environment. Perhaps, such a being could be "in-charge" of our universe, responsible for creation and management of new worlds and also destruction of planets and celestial bodies. Perhaps we've just been calling such a being as "God", and God is nothing supernatural, but rather just science, and can be explained with a theory similar to any other theory of physics.

\section{God in Religion}

Hinduism has over 33 million gods, each "responsible" for a particular aspect of the Universe and a particular aspect of our lives too. The principal Hindu deities are Brahma - The Creator, Vishnu - Protector and Shiva - Destroyer respectively. Likewise, the Goddess Saraswati for Knowledge \& Wisdom, Lakshmi - Fortune \& Wealth and so forth. Christianity, on the other hand, is monotheistic, describing one God who is almighty, all-powerful and all-knowing, propagated by Jesus Christ. Islam is also a monotheistic religion, formed out of the teachings of the Prophet Muhammed. Similarly, Buddhism, Jainism, Zoroastrianism and most other religions are formed by the teachings of certain "enlightened" saints, such as the Buddha, Mahavira or Zoroaster. Other religions consider the worship of nature and natural elements, such as fire. While religion has become more of a tool for building political strategies, the values taught by any religion are more or less the same. Even in religion, everything points to an "Almighty" who is capable of controlling anything in this world and our Universe. It is understandable as to why the people of ancient times came up with the idea of God. The idea of God popped into existence all by itself seem strange is due to their surroundings. It also goes with an irony that someone had to have created it. Hence, they credited the creation of their surroundings, nature and the entire Universe to an omnipotent being - The Creator or God. 
With the growth of modern science, which is strongly based on logical and rational thinking, modern scientists denied the existence of a God. In the name of "supernatural", they declared the "Special Creation Theory" obsolete, because it seemed too unnatural. Moreover, with the growth of modern science, came the growth of an atheistic population. However, there is a possibility that if a God exists, he or she could well be within the laws of science and not a supernatural being.

\section{Higher Spatial Dimensions}

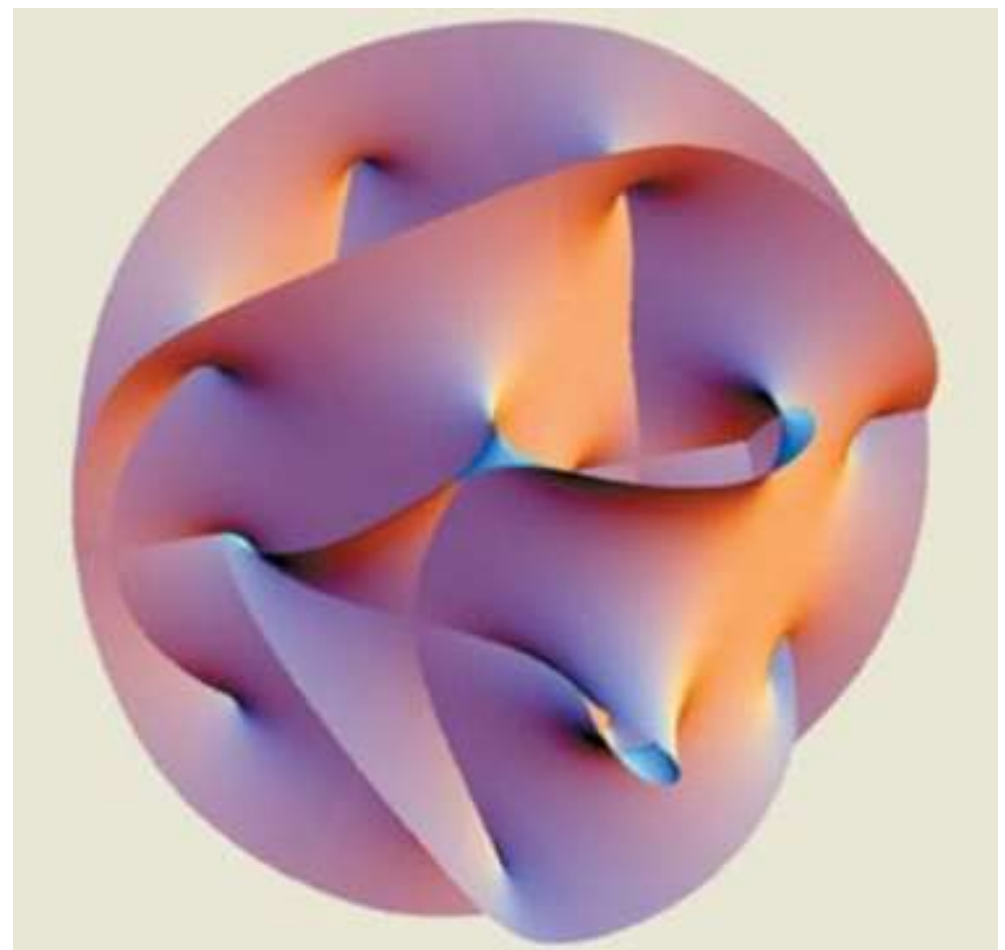

Fig.1 - The Calabi-Yau Manifold Source: Secondary

Modern scientists, in their quest for finding the truths of Universe, proposed several theories explaining the functioning of everything, from quantum particles to quasars. The higher spatial dimensions were proposed as an attempt to support theories such as the String Theory, Superstring Theory and M-Theory. Interestingly, the String Theory requires at least 10 dimensions to support its existence. In fact, scientists believe that there are more than 10 dimensions, but they are unstable and have collapsed and are too small. That doesn't mean they don't exist. There are several modern theories require higher dimensions to exist, so scientists believe that they have to exist and are constantly on the lookout for proof for their existence. The reason being, when we walk on a rope, we cannot move side-ways on it, only forwards and backwards, as it is too thin. However, an ant on the rope can move 
side-ways as well as forwards and backwards. This is the basic idea of the Theory of Compactification of higher dimensions. This theory is a generalized form of Kaluza-Klein Theory, which was developed by Kaluza and Klein in 1926. It states that higher dimensions are not accessible to us because they are "compactified" (shrunken) and has taken the form of a Calabi-Yau manifold. This gives us a good possibility of existence of higher dimensions. Furthermore, the higher dimensions are theorized to include more and more parallel universes with different conditions and physical laws until a final dimension, which includes everything that is fathomable by humans.

The 10 dimensions, as required by the String Theory are as follows:

\section{a. The First Dimension}

As written by Euclid in his book Elements, "a line is breadth-less length". The first dimension comprises only length and no height (Euclid, 1956).

\section{b. The Second Dimension}

The second dimension adds breadth to the equation. This makes the existence of $2 \mathrm{D}$ geometric shapes like polygons possible. Moreover, we have control over this dimension too, since one can draw and manipulate geometric figures.

\section{c. The Third Dimension}

The third dimension is represented by depth or height. Every third dimensional object occupies space, due to its "volume". This is the dimension we are all familiar with, wherein one has complete understanding of above three basic dimensions, as the world we live in is three dimensional.

\section{d. The Fourth (Temporal) Dimension}

The fourth dimension is Time, which by itself is a temporal dimension as it is a way of measuring physical change. Moreover, in this dimension we have no control over the progression of time, but one can perceive time as it moves in one direction towards the future. In furtherance, we do not have the ability to control time and make it move backwards. Therefore, time is not a special dimension, which makes it unique among the other dimensions, which are spatial. Hence, one can say that this dimension is present along with other dimensions, as time is required for entropy. 


\section{e. The Fifth Dimension - Entering Hyperspace}

Dimensions after the fourth are known as hyperspace. The hyperspace is a popular concept portrayed in many science-fiction movies. However, with the evolution of String Theory and development of path towards a "Theory of Everything", hyperspace is hypothesized by many recent studies and modern-day scientists across the globe. Though hypothetical, it is widely believed. The fifth dimension is where the idea of parallel universes or "Multiverse" arises. It is hypothesized that in the fifth dimension, one could see a parallel universe that is slightly varied from our own, yet having the same origin and same physical laws as ours. This could also mean that a person who has control over the fifth dimension could travel between various universes, compare different universes and possibly even travel forwards or backwards in time.

\section{f. The Sixth Dimension}

In the sixth dimension, we would hypothetically able to see multiple parallel worlds having slight variations from ours, yet having the same origins and physical laws. It is believed that by mastering the fifth and sixth dimensions, one could have complete control over time.

\section{g. The Seventh Dimension}

The seventh dimension opens the possibility of existence of universes having varied origins and physical laws as compared to our own. This results in universes having a vast range of differences as opposed to parallel universe in the fifth and sixth dimensions with minor differences. Theoretically, a person who has mastered the seventh dimension could have control over multiple universes both similar and dissimilar to our own. Technically, they could be a "god" to us.

\section{h. The Eighth Dimension}

In the eighth dimension, one could witness the various possibilities of universes each having different origins and different physical laws. In other words, one could see all the parallel, branching universes which progress in different ways. Hence, a person who has mastered the eighth dimension would have control over multiple varied universes.

\section{i. The Ninth Dimension}

The ninth dimension consists of all universes with all possible origins and physical laws, wherein their histories and futures branching out into infinite possibilities. Therefore, someone who has mastered the ninth dimension could have control over 
various universes with all possible origins and laws and also their infinite branches each having different futures.

\section{j. The Tenth Dimension}

The tenth dimension consists of everything that can ever be imagined by humans. It is the final dimension with respect to our abilities of imagination. Beyond this, we in the third dimension will not be able to fathom. Since the tenth dimension consists of everything, one who has mastered this dimension can control everything. In this every instance and possibility of every universe would be under their control. He or She who has mastered the final dimension would be god himself / herself.

\section{Even Higher Dimensions}

The tenth dimension is final only with respect to the abilities of our mind. There may be higher dimensions after the tenth probably eleventh, which will not be able to comprehend. However, it is also theorized that a universe with dimensions higher that of tenth or eleventh would be unstable and would collapse. So, in theory, there doesn't seem to be a definite limit to the number of hyper spatial dimensions.

\section{Discussion - God in Science}

The aforementioned explanations of ten dimensions are only hypothetical. The ten dimensions have been proposed only because it was needed to support the 'Superstring Theory'. There is no evidence for the contents of hyper spatial dimensions. However, one thing that is certain is that higher dimensions mean more universes and more possibilities respectively. We believe in the analogy of God to be someone or something supernatural and all-powerful, as if having control over the universe is not possible scientifically. Theoretically, God could be just someone in higher dimension state. For instance, God present in tenth dimension has absolute control over functioning of entire universe. Moreover, God may have the hyper spatial resources to create celestial bodies in our universe like we have bricks to make buildings. God may also have capabilities to destroy objects in our universe. The so-called "miracles" could just be a higher dimensional power at work.

\section{Suggestion}

The existence of God is always a paradox or abstract and subject of argument. However, with a deeper understanding of dimensions based on 'String Theory' and its higher order dimensions it provides a possible condition of existence of such powerful being unimaginable by humans. This chapter conceptually highlights certain aspects in accordance with dimensions. A holistic research needs to be conducted on true scriptures, whichever validated and accepted by global historians and failures of logical reasoning with many unanswered events and / or aspects by 
scientific communities until now are the gateways to support and understand supernatural avenues in such higher dimensions, which could possibly prove existence of God.

\section{Conclusion}

Ever since rationalism took over the world, we followed the path of logical and rational science, which failed to explore anything that seemed illogical to us and dismissed it as a hoax. Moreover, we failed to utilize intuition and creativity to make discoveries or inventions, thinking that only facts and logic can help progress science. However, creativity is required for discovering facts, based on which we can build our knowledge about science. Hence, this rational mindset has hindered the progression of human civilization. Our belief in God is what makes us wonder about something beyond this material universe. Also, it is what kindles creativity in us and keeps all in this world with curious and fearful of unknown. Hence, we must understand that science does not mean that one has to follow atheistic ideologies, but has to be open-minded to analyze everything, logical or not, in terms of science, instead of dismissing it as a myth or hoax. These approaches discussed in this chapter could possibly answer to questions of paradoxes, wherein, one can conclude that an action resulting in paradox would just not be allowed by the natural law of universe defined by someone or something in higher dimensions. Perhaps, someone whom we would probably refer to as 'God' is the one without terming the aforesaid as supernatural.

\section{References}

Einstein, A. (1921). Relativity: The Special and General Theory, Translated by Lawson, R. W., Henry Holt \& Company, New York, USA, 168p.

Euclid (1956). The Thirteen Books of the Elements, Translated by Heath, T. L., Vol.1, Dover Publications Inc., 443p.

Euclid (1956). The Thirteen Books of the Elements, Translated by Heath, T. L., Vol.2, Dover Publications Inc., 464p.

Kaluza. T. (1921). On the Unity Problem of Physics, Headquarters, Preuss, Akad, Wiss, Berlin, pp.966-972.

Klein, O. (1926). Quantum Theory and Five-Dimensional Relativity, Magazine for Physics, Vol. 37, No.12, pp.895-906. 
Holistic Research Perspectives Vol.5

\section{Webliography}

https://en.wikipedia.org/wiki/Kaluza\%E2\%80\%93Klein_theory

https://en.wikipedia.org/wiki/Theory_of_relativity

https://www.pbs.org/wgbh/nova/article/imagining-other-dimensions/

https://www.universetoday.com/48619/a-universe-of-10-dimensions/ 